Mens

Revue d'histoire intellectuelle et culturelle

mens

Michel Bock et François Charbonneau (dir.). Le siècle du

Règlement 17 : regards sur une crise scolaire et nationale, Sudlbury, Éditions Prise de parole, 2015, 460 p.

\title{
Julien Massicotte
}

Volume 18, numéro 2, printemps 2018

URI : https://id.erudit.org/iderudit/1066264ar

DOI : https://doi.org/10.7202/1066264ar

Aller au sommaire du numéro

Éditeur(s)

Centre de recherche en civilisation canadienne-française

ISSN

1492-8647 (imprimé)

1927-9299 (numérique)

Découvrir la revue

Citer ce compte rendu

Massicotte, J. (2018). Compte rendu de [Michel Bock et François Charbonneau (dir.). Le siècle du Règlement 17 : regards sur une crise scolaire et nationale,

Sudbury, Éditions Prise de parole, 2015, 460 p.] Mens, 18(2), 117-123.

https://doi.org/10.7202/1066264ar d'utilisation que vous pouvez consulter en ligne.

https://apropos.erudit.org/fr/usagers/politique-dutilisation/ 
efficacité les enjeux essentiels de ce corpus de lettres. En somme, nul doute que cet ouvrage ne manquera pas d'intéresser un lectorat varié, depuis les psychanalystes qui y trouveront un terrain d'étude exemplaire jusqu'aux historiens de toute sensibilité qui apprécieront la mise à disposition de cette source unique pour l'étude de la société québécoise ainsi que de la vie religieuse dans l'entre-deux-guerres. Cette parution réjouira également les (encore trop) rares historiens et historiennes du corps et de la sexualité au Québec en leur offrant un matériau de choix pour leurs enquêtes, mais surtout en contribuant activement à la reconnaissance comme au développement de leurs champs de recherche.

- Alexandre Klein

Université d'Ottawa

\section{Michel Bock et François Charbonneau (dir.). Le siècle du Règlement 17 : regards sur une crise scolaire et nationale, Sudbury, Éditions Prise de parole, 2015, 460 p.}

Dans cet ouvrage collectif faisant suite à un colloque qui s'est tenu en automne 2012 et qui portait sur le centenaire de l'adoption du Règlement 17, les historiens Michel Bock et François Charbonneau se donnent le mandat de réexaminer ce " moment déterminant de l'expérience collective des Franco-Ontariens» (p. 24). Le livre reprend là où les travaux de plusieurs chercheurs, dont Robert Choquette et Gaétan Gervais - deux sources d'inspiration manifestes émaillant les pages de ce collectif — avaient laissé la question.

L'ambition des directeurs de l'ouvrage se traduit par la dualité de la réflexion qu'ils proposent en introduction. Le premier ordre de réflexion, se situant dans le prolongement des travaux antérieurs de ces deux directeurs, se veut général et synthétique : on cherche à comprendre l'influence du Règlement 17 sur l'histoire subséquente de la communauté franco-ontarienne et sa portée dans l'histoire canadienne-française en général. Un second ordre de réflexion porte, quant à lui, sur la pluridimensionnalité des éléments en jeu lors de 
cette crise scolaire et nationale. L'examen de ces éléments se voudra plus empirique, descriptif et exploratoire. On privilégiera, dans plusieurs des études de ce livre, l'explication et l'illustration, ce qui est sans doute l'une de ses forces, cette approche seyant à un objet d'étude de la sorte.

Le livre compte cinq sections, qui explorent les différentes facettes du Règlement 17 et de ses répercussions sur l'Ontario francophone et l'ensemble du Canada français des premières décennies du $\mathrm{xx}^{\mathrm{e}}$ siècle; on y aborde tour à tour la réaction canadienne-anglaise, la résistance émanant de différents milieux, l'émergence d'une réflexion politique et constitutionnelle autour du règlement, l'appropriation du règlement dans le monde littéraire et théâtral et, finalement, la transformation $\mathrm{du}$ « champ intellectuel canadien-français » qui lui a succédé.

La première section de ce volume porte sur la réception du Règlement 17 au Canada anglais. Elle aborde l'attitude de la communauté irlandaise face au règlement, celle de la presse anglophone et d'individus influents à l'époque, tels que Napoléon-Antoine Belcourt et William Henry Moore. Le texte de Sylvie Lacombe, dans cette section, constitue sans conteste l'une des clés interprétatives nécessaires pour comprendre plusieurs des études dans ce recueil. Lacombe reprend un thème qu'elle explore depuis plusieurs années, soit la comparaison des configurations idéologiques canadienneanglaise et canadienne-française. Elle présente une lecture de la réaction de l'intelligentsia canadienne-anglaise face au Règlement 17, réaction découlant largement de l'omniprésence de l'impérialisme britannique à l'époque. Cette idéologie constitue le cadre de référence par excellence des élites canadiennes-anglaises, et l'on remarque à plus d'un endroit dans l'ouvrage des exemples où des élites, francophones comme anglophones, se servent de l'idéologie impérialiste comme moyen pour critiquer tout ce que représente le Règlement 17. Hans-Jürgen Lüsebrink cite l'exemple de William Henry Moore, un anglophone qui se porta à la défense des Canadiens français touchés par le règlement, dénoncé « comme étant contraire à l'esprit britannique et à la conception de la nation canadienne " 
(p. 98). Cette première section de l'ouvrage avance donc le constat suivant : l'impérialisme constitue le système référentiel dominant à l'intérieur duquel prend place le Règlement 17, un fait souvent négligé par rapport à l'importance consentie à la référence nationaliste de l'époque. La première section dévoile ce contexte de confrontation à l'intérieur duquel se déroulent les luttes autour du Règlement 17, et la nécessité, pour les acteurs individuels et collectifs, d'adopter le langage et le discours des forces dominantes de l'époque.

La seconde section de ce volume aborde les multiples facettes de la résistance face au Règlement 17, illustrant toute la complexité de la mobilisation d'organisations et de groupes franco-ontariens et canadiens-français de l'époque. Il s'agit là manifestement de l'une des sections fortes du livre. On y ausculte tour à tour les réactions du journal Le Devoir, de l'Association canadienne-française d'éducation d'Ontario (ACFEO), de l'Union Saint-Joseph, des francophones du sud-ouest de l'Ontario, tout en faisant un examen approfondi de la question des écoles bilingues. Gratien Allaire cite l'historien Gaétan Gervais, qui mettait en garde contre une simplification à outrance des tenants et aboutissants de la crise du Règlement 17 : "La lutte scolaire autour du Règlement XVII s'explique par la convergence de plusieurs antagonismes : l'opposition idéologique entre nationalistes et impérialistes; la crainte des Ontariens anglo-protestants devant l'immigration franco-catholique; les vieilles rivalités entre les orangistes (Irlandais protestants) et les catholiques; les luttes cléricales entre catholiques français et irlandais; la divergence d'intérêts politiques partisans » (p. 161-162). Étant donné le statut qu'occupe le Règlement 17 dans l'imaginaire franco-ontarien, on pourrait en effet supposer un engagement unanime de la part des différents acteurs collectifs. Or, suivant en cela la précaution de Gervais, plusieurs de ces études nous proposent plutôt une lecture nuancée de la situation. Dans son étude sur l'Union Saint-Joseph, Pierrick Labbé signale l'oscillation de cette organisation entre la propagande nationaliste et la critique timide des milieux anglophones favorables au règlement, notamment le clergé irlandais. Le texte de Serge Dupuis sur l'ACFEO 
est de même mouture : il montre clairement l'existence parallèle des différentes voies qu'emprunte la résistance des milieux francoontariens, alternant entre la résistance et la persuasion populaire. Revenons au texte d'Allaire. Il est sans doute l'un des seuls textes du recueil à prendre au sérieux l'aspect "scolaire " de la crise. Allaire n'hésite pas à y approfondir la place qu'occupe l'école dans l'imaginaire franco-ontarien de l'époque, de même que les détails et les subtilités de l'école française, anglaise et bilingue dans l'Ontario du début du $\mathrm{xx}^{\mathrm{e}}$ siècle. Cette étude constitue un apport essentiel pour comprendre l'ensemble de la problématique traitée dans l'ouvrage.

La troisième section de cette publication concerne les questions d'ordre politico-juridique. On y examine tour à tour la place du Règlement 17 telle que débattue devant le Parlement, l'engagement de Thomas Chapais dans la crise ainsi que les aspects constitutionnels tournant autour du règlement. La contribution de François Charbonneau revient sur le thème du nationalisme et de l'impérialisme, l'un des fils conducteurs du livre. Ce texte présente un Canada en pleine redéfinition à l'époque, ou en pleine crise. Ce constat devient prétexte pour examiner avec force détails la question référentielle canadienne, à partir de la perspective d'une minorité linguistique, mais également d'une communauté historique fondatrice. Pierre Anctil, dans son texte portant sur Le Devoir et le Règlement 17, s'enquiert de la nature du rapport unissant les « deux peuples fondateurs ». Charbonneau, adoptant une perspective semblable, résume le fossé qui divise les deux communautés autour de cette question du pacte fondateur : " [D]isons-le crûment : un seul des deux "pactisants" semble avoir été mis au courant du fait qu'il y a eu pacte... " (p. 277). Le juriste Pierre Foucher présente, quant à lui, une analyse fort pertinente, centrée sur l'aspect juridique du Règlement 17 et sur un effort de contextualisation de la question dans un cadre canadien plus vaste. Cet effort est certainement l'un des aspects les plus importants de cette étude, et Foucher est (malheureusement) l'un des seuls à procéder de la sorte dans l'ouvrage, en situant le Règlement 17 dans le contexte des crises scolaires que traversait le 
Canada français : il n’hésite pas à proposer des éléments comparatifs (et éclairants) tirés de l'histoire acadienne ou de l'Ouest, par exemple.

La quatrième section est consacrée à la présence du Règlement 17 dans les sphères littéraire et artistique, révélant ainsi le legs du règlement dans la conscience collective franco-ontarienne. Les trois textes de cette section portent sur la présence du règlement en littérature, sur l'importance de la salle Sainte-Anne d'Ottawa et sur la représentation théâtrale de la crise. Bien que cette partie avance des éléments d'analyse nouveaux, elle constitue sans doute la partie la moins " essentielle ", du simple fait que plusieurs autres articles du collectif, dès les premières pages, mentionnent l'importance du Règlement 17 dans l'imaginaire franco-ontarien. Dès lors, les apports de cette section, quoiqu'importants, se voient quelque peu dilués.

La dernière partie, intitulée « La crise scolaire et la reconfiguration du champ intellectuel canadien-français ", contient trois des contributions les plus importantes de l'ouvrage, soit la conclusion de Bock, qui doit être lue à la fois comme une synthèse de ce recueil et comme un texte prospectif qui arpente les pistes de réflexion à venir, ainsi qu'une chronologie et une bibliographie détaillée du Règlement 17, signées toutes deux par Bock et Simon-Pierre ChaplainCorriveau. On soulignera ici l'intérêt de ces deux derniers documents pour toute la communauté de chercheurs, ouvrant à une meilleure compréhension et à une plus juste connaissance de cette crise, mais également du passé canadien-français dans son ensemble.

Le texte de Bock, sans surprise, suivant les réflexions des Dumont et Gervais, énonce ses propres considérations sur le nationalisme du chanoine Groulx et aborde la question du Règlement 17 dans le contexte plus large de l'évolution de la référence nationale canadiennefrançaise durant la première moitié du Xx ${ }^{e}$ siècle. Cette étude montre bien comment la crise du Règlement 17 devient un moment catalyseur pour la mise en forme d'une référence collective dont les principaux éléments sont la religion, la langue et l'appartenance nationale. L'historien indique que cette crise se déroule à l'intérieur d'une dynamique foncièrement religieuse : tensions avec le clergé irlandais, 
subordination à la hiérarchie romaine, etc. Les années 1920 marquent un tournant dans la crise nationale. L'ACFEO, principale porte-parole, selon Bock et plusieurs auteurs de ce collectif, des intérêts des FrancoOntariens dans le contexte de la crise, s'efface à partir de 1926 au profit de l'Ordre de Jacques-Cartier, dont les tactiques et la vision sont quelque peu différentes, moins axées sur la confrontation directe, notamment. Le changement de garde révèle, aux yeux de Bock, l'amorce d'un tranquille déclin de ce «nationalisme groulxiste », qui connaîtra sa conclusion logique durant les années 1960.

Une des limites de ce livre, que partage toutefois, il importe de le signaler, la majorité des études portant sur les crises scolaires francophones au Canada, est le peu d'attention accordée à l'institution scolaire elle-même. Le collectif, dont le sous-titre est "Regards sur une crise scolaire et nationale ", présente la question scolaire comme étant largement subordonnée à la question nationale. Mon commentaire ne cherche pas, évidemment, à faire porter le blâme aux chercheurs qui ont contribué à l'ouvrage. D'une part, il est vrai que, sur le plan des débats qui l'entouraient, cette crise scolaire constituait d'abord et avant tout une crise nationale, culturelle, religieuse, linguistique et politique. La question de ce que représentait l'école à l'époque, de ce qui établissait son rôle, sa fonction sociale, n'est pratiquement pas évoquée. Seul le texte d'Allaire aborde de manière substantielle le rôle de l'éducation et de l'école. Le livre de Bock et de Charbonneau a tout de même accompli un travail de défrichage et de synthèse nécessaire. Le prochain chapitre de cette réflexion - et ce tome en a certainement préparé le terrain — sera probablement d'analyser la place qu'y occupent l'école et l'éducation.

En somme, ce collectif sur la crise du Règlement 17 réussit à accomplir son mandat initial, soit de réexaminer un événement de l'ordre du mythe, un récit d'origine en quelque sorte, pour en approfondir ses différentes dimensions. Il contribue, de façon rigoureuse et cohérente, à l'avancement des connaissances sur la crise du Règlement 17. Les études portant sur les francophonies canadiennes connaissent un certain renouveau; cet ouvrage, qui marie bien les 
contributions des chercheurs établis à celles des chercheurs émergents, en est une preuve. On évoque souvent les crises scolaires comme fondatrices dans l'histoire du Canada français, et chacune des "francophonies spécifiques ", hormis le Québec, possède sa propre version de la crise scolaire, avec des incidences de rupture et d'autonomie similaires à ce qu'a connu l'Ontario francophone. Cet ouvrage éclaire de façon neuve et essentielle cette question en Ontario. Souhaitons que cet effort intellectuel constitue l'amorce d'un examen approfondi, à plus grande échelle, de la problématique pour toutes les francophonies canadiennes.

— Julien Massicotte

Secteur des sciences humaines Université de Moncton, campus d'Edmundston 